\title{
IaaS, PaaS, or SaaS? The Why of Cloud Computing Delivery Model Selection - Vignettes on the Post-Adoption of Cloud Computing
}

\author{
Frederik Wulf \\ TU Dresden \\ frederik.wulf@mailbox.tu-dresden.de \\ Markus Westner \\ OTH Regensburg \\ markus.westner@oth-regensburg.de
}

\author{
Tobias Lindner \\ TU Dresden \\ tobias.lindner@mailbox.tu-dresden.de \\ Susanne Strahringer \\ TU Dresden \\ susanne.strahringer@tu-dresden.de
}

\begin{abstract}
Most large-scale organizations adopted Cloud Computing (CC) on a company level in recent years. Managers now face the challenge to appropriately implement CC "operationally", i.e., for information systems (ISs). We refer to this as post-adoption, addressing the extent of technology usage after adoption. Specifically, managers need to choose among the CC delivery models Infrastructure-as-a-Service (IaaS), Platform-as-a-Service (PaaS), and Software-asa-Service (SaaS). We differentiate the determinants of this post-adoption decision for IaaS, PaaS, and SaaS. Based on this analysis, we derive criteria that guide managers' delivery model selection: Adopt 1) IaaS for ISs requiring flexibility and reduced time to market, 2) PaaS to access specialized resources, and 3) SaaS to focus on core competencies.

Moreover, we analyze the impact on the CC strategy and postulate them as recommendations: I) acknowledge the interplay between governance and time-to-market, II) realize cost savings on company level, and III) consider strategically important ISs for CC.
\end{abstract}

\section{Introduction}

Cloud Computing (CC) has become a widely used technology at most large-scale companies. Three out of four companies already made the strategic decision to use CC on a company level (adoption) [1].

During a pilot case-study in a large-scale company with $>25$ bn USD revenue in 2019, we identified two relevant patterns [2]: Firstly, the company adopted all CC delivery models on a strategic level (adoption). Secondly, after the strategic decision to use $\mathrm{CC}$ on a company level (adoption), the pilot company currently deals with the challenge of operatively adopting $\mathrm{CC}$ on the level of individual Information Systems (ISs). We refer to this IS-level implementation of CC as postadoption, which describes how "technology is actually used" [3, p. 363] after company-level adoption.

Considering that the pilot case study's findings [2] may apply to a broader set of corporations, we investigated the phenomena of CC post-adoption in a multiple case study of large-scale companies across different industries.

In this paper, we investigate three CC delivery models [4]: Infrastructure-as-a-Service (IaaS) provides the customer an environment to host ISs, Platform-as-aService (PaaS) provides an environment for IS development, and Software-as-a-Service (SaaS) provides a ready-to-use IS. As the CC service level scope varies by delivery models [5], the challenge for managers is to select the appropriate delivery model for an IS under consideration.

While various research papers investigated what determines CC adoption on the company-level, research has not yet differentiated the IS-level post-adoption determinants by delivery models. The lack of such an understanding of delivery model-specific post-adoption determinants constitutes a research gap. It serves as a prerequisite to deduce decision criteria for managers to select the appropriate delivery model in IS-level postadoption sourcing decisions. Moreover, the implications from practical experiences with CC IS-level postadoption for the company-level CC strategy have not yet been analyzed. Hence, our research questions (RQ) address these research gaps.

RQ1: What are the CC post-adoption determinants of IaaS, PaaS, and SaaS on the level of individual ISs?

RQ2: What are the decision criteria for selecting the appropriate CC post-adoption delivery model for individual ISs?

RQ3: What are the strategic implications of IS-level $\mathrm{CC}$ post-adoption? 
The RQs exhibit a strong practitioner focus, constituting a practice-oriented research design [6]. Alike, we present our findings accordingly in the form of case vignettes, providing "rich stories" and "unique insights" [7], to ensure practice-oriented data analysis [6].

The contribution of this paper is twofold: Firstly, the paper contributes to theory in investigating RQ1 by analyzing differences across delivery models, potentially explaining why, at times, research on $\mathrm{CC}$ adoption comes to diverging conclusions. Secondly, the results provide practical guidance for practitioners that a) consider which delivery model to use in a postadoptive setting (answered in RQ2), and b) are interested in strategic implications for practice [6] derived from the key learnings of real-life implementations of $\mathrm{CC}$ in large-scale companies (RQ3).

The rest of the paper is structured as follows: Section 2 provides background on the research subject. Section 3 outlines the methodology applied and the data foundation of this paper. Section 4 presents our findings, followed by a discussion in Section 5. Section 6 concludes the paper.

\section{Research background}

Corresponding to the paper's practitioner focus, we restrict the research background to the essential knowledge to understand the conducted analysis. We provide an introduction into the research background by summarizing the knowledge on CC adoption determinants that is not differentiated by delivery model as a starting point for analyzing RQ1. After that, we outline the results from our pilot study that guided us to identify the RQs.

Regarding RQ1, as outlined in this paper's introduction, the differentiation by delivery model found little consideration in the literature so far. On CC in general, Schneider \& Sunyaev [5] provide an overview of empirically tested variables, so-called "determinants", with consistent findings grouped into asset (i.e., the IS), technology, client, individual, CSP, and environment characteristics which influence the sourcing decision. Thereof, we consider the IS and technology characteristics as relevant background information because they are likely to exhibit variations between the delivery models. The main findings relevant for the paper at hand are: Regarding the IS, cost savings (e.g., [8]) positively and strategic importance (e.g., [9]) negatively influence the decision to use CC. Access to specialized resources (e.g., [8]), flexibility (e.g., [10]), and reduced time to market (e.g., [8]) positively influence CC adoption. Moreover, some evidence suggests a positive influence of the ability to focus on core competencies for CC adoption (e.g., [10]). Security risks (e.g., [11]) are negatively associated with $\mathrm{CC}$ adoption, i.e., contribute to a decision not to use $\mathrm{CC}$ at all. The appendix provides definitions for these determinants.

In a pilot study, we identified different patterns in adopting the three CC delivery models [2]: The company adopted SaaS driven by business-demand. Adoption included the provisioning of the specific IS as requested from the business side. The adoption of IaaS and PaaS, however, was initially an IT-driven offering. We observed the pattern that the adoption process included two steps: First, the company integrated the cloud service provider (CSP) in the information technology (IT) landscape (adoption). Then, the company adopted the CSP's Iaas/PaaS offerings for IS development and hosting (post-adoption). We distinguish the initial (strategic) "adoption" on the company level from the (operational) "post-adoption" of deciding to use a specific CC delivery model for an individual IS. Consequently, this raised our interest in whether post-adoption determinants depend on the delivery model, leading to RQ1.

\section{Research methodology and data foundation}

Our interest in investigating the research questions stems from a prior case-study that can be considered a pilot study in terms of Gable [12], focusing on analysis by description. To increase the generalizability of the identified patterns, the next research step is to conduct a pattern analysis across company contexts within a casestudy based on multiple companies [12].

To find participants to share their experiences with the implementation of CC delivery models, we invited contacts from a prior study for participation. Therefore, the authors knew that the invited participants had relevant professional experience with the implementation of CC in large-scale organizations. Following the sampling approach of planned opportunism [13], where case selection depends on "research interest [...] and explicit opportunities" [13, p. 165], we sent 25 invitations, of which 10 interviews resulted. One additional interview resulted from a personal contact of one of the authors that agreed to contribute to the study. We excluded three interviews from analysis, as participants could not dwell on specific IS-level implementations or acted as CSP itself.

Table 1 provides an overview of the participants of the conducted study. We anonymized the participants' names and companies, as requested by the participants. All participants work within large-scale organizations (more than 50,000 employees and more than 2 billion Euros in revenues) headquartered in Germany. The participants have more than five years of professional experience in the field of IT or related areas (except for $V$ 's participant with two years). All participants worked 
Table 1. Overview of study participants

\begin{tabular}{|c|c|c|c|c|}
\hline Index & Implementation & Delivery model & Role title(s) & Industry \\
\hline$V_{1}$ & Service platform extension & laaS & Cloud Technical Lead & Financial Services \\
\hline$V_{2}$ & Data lake & laaS & Cloud Architect & Automotive OEM \\
\hline$V_{3}$ & $\begin{array}{l}\text { Development environment } \\
\text { service-pipeline }\end{array}$ & laaS & $\begin{array}{l}\text { Chief Information Security } \\
\text { Officer }\end{array}$ & Financial Services \\
\hline$V_{4}$ & Delivery tracking system & Paas & $\begin{array}{l}\text { IT Expert Cloud Center of } \\
\text { Competence }\end{array}$ & Logistics \\
\hline$V_{5}$ & Product comparison system & PaaS & Director of Analytics & Retail Trade \\
\hline$V_{6}$ & $\begin{array}{l}\text { Truck tracking and guiding } \\
\text { system }\end{array}$ & PaaS & Global Category Buyer Cloud & Chemicals \\
\hline$V_{7}$ & HR system & SaaS & IT Specialist & Automotive OEM \\
\hline$V_{8}$ & Digital signature system & SaaS & $\begin{array}{l}\text { Transformation Manager } \\
\text { Digitalization }\end{array}$ & $\begin{array}{l}\text { Automotive } \\
\text { Supplier }\end{array}$ \\
\hline
\end{tabular}

in different companies so as not to overrepresent the experiences of a single company.

We conducted the interviews in April/May 2020 in a semi-structured manner around three themes: a) context setting on the company's overall CC usage and the study participant's role, b) mini-case description of the IS where $\mathrm{CC}$ was adopted, c) as well as mini-case analysis regarding the determinant factors for $\mathrm{CC}$ delivery model choice. The interviews lasted one hour, and at least two of the authors were present in the eight interviews. We recorded and transcribed the interviews if the participant agreed and took detailed notes otherwise. The documentation and analysis followed the four-eyes principle. The authors coded the statements from interviews individually and compared the results afterward. Arising conflicts considering the interpretations were raised and resolved among the authors.

Evaluating reasons for choosing a particular delivery model required the correction of different language usage and conventions for yet the same theme. We, therefore, mapped the reasons stated by the participants towards a taxonomy of $\mathrm{CC}$ adoption determinants developed for IT outsourcing and CC adoption from Schneider \& Sunyaev [5] that integrates the results of prior research on IT outsourcing [14], [15]. By doing so, we aim to ensure consistency with prior research in the field.

For the presentation of the study's results, we choose the format of results presentation vignettes for each case company. Niemimaa \& Niemimaa [16, p. 573] have defined vignettes "as short but detailed descriptions, [providing] vivid, authentic, and evocative accounts of the events and [seeking] to increase the truthfulness, plausibility, and credibility of the findings." It is not new to use the form of case vignettes for describing the results of empirical research: Huang Chua \& Myers [17] use four vignettes to present their findings on social control in IS development. Kotlarsky et al. [18] use five vignettes to present findings in the context of IS offshoring projects.

\section{Case vignettes on $\mathrm{CC}$ post-adoption}

\subsection{Results overview}

Table 2 shows a results overview of the conducted interviews and the implications the different determinants have on CC post-adoption, compared to the CC adoption results of Schneider \& Sunyaev [5].

Amongst participants, cost savings found little consideration on IS level, which contradicts existing literature investigating $\mathrm{CC}$ adoption. Alike, the strategic importance of the considered application is a driver for CC post-adoption for IaaS and PaaS, which previously yielded a negative influence on CC adoption [5]. Participants considered reduced time to market as a decisive factor of CC across all delivery models. Additionally, flexibility is a strong driver for IaaS postadoption. In contrast, access to specialized resources supports the post-adoption of PaaS, and focus on core competencies promotes the post-adoption of SaaS. The topic of security risks reveals ambiguity amongst the participants, mentioning positive and negative implications for the post-adoption across delivery models.

\subsection{Case vignettes on IaaS post-adoption}

4.2.1. "It's freedom" - Service platform extension $\left(V_{1}\right)$. The case company is a global financial service provider offering insurance and investment products in various countries. Overall, the aim is to distribute a third of the workload each to on-premise infrastructure and to the two IaaS/PaaS CSPs they contracted, managing operations of CC from the central IT function of the company. Despite the global footprint of the company, it hosted the product platforms widely distributed and locally within each country to accommodate local regulations and consumer preferences in the country of operation. The company's study participant acted as 
Table 2. Results overview from case vignettes

\begin{tabular}{|c|c|c|c|c|c|}
\hline Dimension & Determinant & laas & Paas & SaaS & Comparison to [5] \\
\hline \multirow[t]{2}{*}{$\begin{array}{l}\text { Information } \\
\text { System }\end{array}$} & Cost savings & $0\left(V_{1}, V_{2}, V_{3}\right)$ & $0\left(V_{4}, V_{5}, V_{6}\right)$ & $\begin{array}{l}0\left(V_{7}\right) \\
+\left(V_{8}\right)\end{array}$ & Inconsistent \\
\hline & Strategic importance & $+\left(V_{1}, V_{2}, V_{3}\right)$ & $\begin{array}{l}+\left(V_{4}, V_{5}\right) \\
0\left(V_{6}\right)\end{array}$ & $\begin{array}{l}-\left(V_{7}\right) \\
0\left(V_{8}\right)\end{array}$ & Inconsistent \\
\hline \multirow[t]{5}{*}{ Technology } & Reduced time to market & $+\left(V_{1}, V_{2}, V_{3}\right)$ & $\begin{array}{l}+\left(V_{4}, V_{5}\right) \\
O\left(V_{6}\right)\end{array}$ & $+\left(V_{7}, V_{8}\right)$ & Consistent \\
\hline & Flexibility & $+\left(V_{1}, V_{2}, V_{3}\right)$ & $\begin{array}{l}0\left(V_{4}, V_{6}\right) \\
+\left(V_{5}\right)\end{array}$ & $0\left(V_{7}, V_{8}\right)$ & Consistent \\
\hline & $\begin{array}{l}\text { Access to specialized } \\
\text { resources }\end{array}$ & $\begin{array}{l}0\left(V_{1}, V_{3}\right) \\
+\left(V_{2}\right)\end{array}$ & $+\left(V_{4}, V_{5}, V_{6}\right)$ & $\begin{array}{l}+\left(V_{7}\right) \\
0\left(V_{8}\right)\end{array}$ & Consistent \\
\hline & $\begin{array}{l}\text { Focus on core } \\
\text { competencies }\end{array}$ & $0\left(V_{1}, V_{2}, V_{3}\right)$ & $\begin{array}{l}+\left(V_{5}, V_{6}\right) \\
0\left(V_{4}\right)\end{array}$ & $+\left(V_{7}, V_{8}\right)$ & Consistent \\
\hline & Security risks & $\begin{array}{l}0\left(V_{1}, V_{3}\right) \\
-\left(V_{2}\right)\end{array}$ & $\begin{array}{l}0\left(V_{5}, V_{6}\right) \\
+/-\left(V_{4}\right)\end{array}$ & $\begin{array}{l}0\left(V_{7}\right) \\
+\left(V_{8}\right)\end{array}$ & Inconsistent \\
\hline
\end{tabular}

Legend: "+" indicates that the determinant had a positive impact on the decision whether to use the CC delivery model, whereas "-" implies an adverse effect and hinders the post-adoption of CC on an individual IS level. "0" indicates that the determinant did not impact decision-making. In the comparison column, we show the findings on individual IS level (post-adoption) compared to existing literature [5] regarding initial CC adoption.

Technical Cloud Lead for half a year and switched from one of the two CSPs that the company uses for IaaS/PaaS.

At the beginning of the COVID-19 pandemic, the company realized that traffic on the digital sales channels increased due to the lockdown measures inaugurated in various countries, making it impossible to serve customers through physical sales channels. These product platforms covered frontend as well as backend systems and are either hosted on-premise or on IaaS. Specifically, in Russia, the traffic on one existing product platform increased significantly. Hence, the existing infrastructure could not support the demand for insurance products to be ordered online. As the product platform was in the middle of its lifecycle, there was no intention to enhance the system while scaling-up its infrastructure.

To accommodate the increased demand of the product platform, the company decided to extend the existing on-premise infrastructure by hosting additional virtual machines (VMs) as CC service, constituting a horizontal scale-up on IaaS. Asked about the rationale behind choosing IaaS for this request to increase the bandwidth of the product platform, interviewee $V_{l}$ stated:

"It's freedom! If we do it in the cloud, we have much more flexibility in how we provision infrastructure. The first copy of the data stays in the local datacenter and, therefore, it is regulatory compliant, and we can do the rest in the cloud. If we wanted to scale-up the on-premise infrastructure, it would take weeks. Also, in the current situation, it is difficult to say how much resources we require in a month from now."
The primary reasons for choosing IaaS, in this case, are the reduced time to market to provide the infrastructure and the flexibility regarding the sizing of the required resources, considering the uncertainty of future demand in these unprecedented times. Online being the dominant sales channel, these advantages mainly played an essential role due to the strategic importance of the IS at hand. Cost savings considerations were negated by the interviewee, stating that costs will mainly depend on the factual load required for the system. PaaS was not chosen in this context because there were none of the functionalities needed to fulfill the business request.

4.2.2. "100 times the amount of data" - Data lake $\left(V_{2}\right)$. The case company discussed is a global automotive original equipment manufacturer (OEM) aiming to increase the development speed of IT-services and use cases by using CC. Further, the OEM aims to increase the standardization of the IT infrastructure by moving to CC. Key executives' target agreements, therefore, include the usage of $\mathrm{CC}$ to incentivize post-adoption. The company pursues a multi-cloud strategy, contracting the two market-leading CSPs and one additional CSP that also functions as a general contractor for some larger IT-projects. The company's study participant acts as a Cloud Architect and is responsible for the network and network concept between on-premise data centers and the different public cloud locations.

The company exhibited slow IT-service provisioning and extensive governance and regulations, leading to long lead times of up to half a year for the provisioning of basic IT-services (e.g., VMs). Considering 
drawbacks, the company tried to avoid these by implementing very light governance when using CC. Project owners could quickly get access to an account that allowed them to provide services themselves as needed but were held fully responsible for the associated costs and compliance to data security.

The company established a data lake to store and combine various sources of corporate data for large, strategic projects. Moreover, the data lake enabled smaller projects to utilize these data sources to develop their use cases. The company ran a big data platform onpremise before, but experienced severe issues to scaleup storage, as the data lake grew:

"It took six months to get an extension on storage, and it was no fun to plan projects with such a lead time. The data lake would not have been possible on-premise as today we are handling 100 times the amount of data than before. Also, access for multiple smaller projects would not have been able on-premise."

Considering the case above, the core determinant for the decision on IaaS is flexibility, especially in terms of scalability. Forming the foundation for multiple projects, the structured collection, and storage of data is of strategic importance. Asked on the reasons for provider selection, the participant pointed out the access to the provider's architects specialized in setting-up data lakes (access to specialized resources). Beyond this, the sourcing choice aims to reduce time to market, driving agility, and innovation in the company. The development speed of new solutions and use cases significantly increased, through bypassing existing legacy structures as well as oppressive corporate governance. In addition to the described benefits, the participant mentions remaining security risks stemming from the implemented light-touch governance.

4.2.3. "Ringfencing the open field" - Development environment service-pipeline $\left(\boldsymbol{V}_{\mathbf{3}}\right)$. The case company is a provider of customer solutions in the financial services industry, and hence, under banking regulations. The study participant is heading the information security department and is therefore deeply involved in the implementation of $\mathrm{CC}$, for which the company follows a multi-cloud approach.

As a consequence of the regulation, the company strictly separates the development environments from the productive systems, both running on heterogeneous infrastructure. Additionally, the development environments need dedication to a specific development project and require separation from one another. Both factors lead to many requests to provision development environments that needed manual checks to avoid uncontrolled growth.

The company decided to adopt IaaS for the provisioning of development environments to provide infrastructure when the need arises timely and to deplete it if the need expires. The chosen solution to the problem was a self-service system enabling developers to provision a standardized development stack themselves in a compliant manner. It integrated role and privilege concepts, audit-trails, geo-segmentation of entities to instantly provisions infrastructure. The system also allowed the business units to provide the relevant environments independently, without help from the IT organization, thus enabling business-managed IT.

"When developers work in the cloud, there is the danger of them being on an open playing field, where they do things that they should not. We needed to ringfence this open playing field to ensure compliance."

The primary determinant leading to IaaS postadoption, in this case, is the flexibility of provisioning and depleting infrastructure. Similarly, it leads to a reduced time to market due to the instant provisioning of infrastructure to developers. Additionally, the company develops all ISs of strategic importance on IaaS, despite being regulatory unable to run most production systems on it.

In contrast to approaches of other case companies, the company integrates technical governance and compliance guidelines in the provisioning of the infrastructure, rather than instructing the employees on their privileges.

\subsection{Case vignettes on PaaS post-adoption}

4.3.1. "We were condemned to succeed" - Delivery tracking system $\left(\boldsymbol{V}_{\mathbf{4}}\right)$. The case company is a logistics provider, mainly in the central European region, but with operations worldwide. The company's study participant works as an expert in its Cloud Competency Center and is responsible for the strategic planning of public-cloud initiatives. Unlike the other researched case companies, the mentioned logistics provider uses a single CSP strategy focusing entirely on one CSP for PaaS/IaaS. The case company differentiates from the others by not employing CC directly, but via a managed cloud provider, a 3rd party provider responsible for managing the processes and operations of CC.

The pilot use case that the interviewee needed to develop during the initial phase of $\mathrm{CC}$ usage was a delivery tracking system of the delivery vehicles as competitors to the company already had implemented similar features. Hence, the vignette concerns a new system development. Implementing this IS required to connect the in-vehicle navigation that tracks vehicle position with route forecasting to estimate the time of arrival (ETA) for a specific delivery. A visualization layer enabled sharing the information with the customer.

At the time of the sourcing decision, the board had already defined and communicated a release date externally. To comply with this tight timeline of only weeks, project managers sourced services from the PaaS 
provider instead of building their proprietary algorithms (e.g., location tracking, ETA forecasting). That sped up the process and was a critical factor for success:

"We had very little time and were condemned to succeed. Using PaaS was without any alternative, we would have never succeeded otherwise, neither onpremise nor building the functionalities on IaaS. On the platform, the required services were readily available and could otherwise never be developed individually in the conventional setting in time."

In the particular case, speed and agility from CC supported the reduction of time to market and to hold the tight deadline. Using predefined functionalities from the CSP, the company leveraged existing capabilities (access to specialized resources) from third party providers. The overall IS was considered of strategic importance to close the gap to competitors in terms of customer service. The participant mentioned the ambiguity regarding security risks. Security considerations were of significant importance "we have developed a 250-page security concept". However, at the same time, the company acknowledged that the CSPs invest significantly more resources in the security of their services than the individual company ever could.

\subsection{2. "We do not have to reinvent the wheel" -} Product comparison system $\left(\boldsymbol{V}_{5}\right)$. The case company is a market-leading retail player, currently conducting a journey from a conventional IT-environment towards using a multi-cloud strategy to avoid lock-in effects. Our interviewee is the Head of Analytics and recently supported the migration of analytics related ISs to CC.

In the analytics team, programmers spent most of their time developing code for analyses that are then run and tested on large data sets. Running analytics on onpremise solutions resulted in peaks of computing power in existing data centers, as our interviewee stated: "95\% of the time, we had unused resources, but during the other 5\% when we ran our analyses, we had too little capacity and created significant issues that got in conflict with other productive systems as controlling or finance." As a result, the company decided to transfer the entire data warehousing to $\mathrm{CC}$ and to use preliminarily pre-trained models and services for analytics (PaaS).

A specific use case for the retail industry was the search and comparison of specific products to the competitors' offerings. That analysis is frequently required when the company considers adding a new product to the portfolio. Therefore, web scraping (retrieving data), automated image recognition (recognizing products), and text analysis (comparing key characteristics) are prebuilt functions that could be sourced directly from the CSP. Potentially, the required functionalities could have been developed individually from scratch, and it might even be the case that these individual models reveal slightly better results. However, it was a question of time and resources to do so:

"For us, it is most important to be capable of quickly testing the applicability of use cases. There is no reason to reinvent the wheel when it comes to services like image recognition, sentiment analysis, or pre-trained forecasting models."

Fast implementation requires to store data in the same environment that also provides the computing power and relevant modules: "We need a holistic dataengineering or machine-learning pipeline, consistently in one system. We need one cloud that allows the automated, consistent, and performant application of analytics use cases."

This vignette reveals that using PaaS predominantly meant to reduce the time to market of newly developed use cases. Additionally, cloud-based solutions allow for full flexibility regarding peaks in needed capacity. The company does not aim to build proprietary analytics algorithms (focus on core competencies) but rather leverages existing resources (access to specialized resources). As a result of this, the company reduces development effort and achieves enhanced innovation through quick testing of ideas with prebuilt services. Finally, integrating analytics systems and cloud-based database hosting allows automation and the exploitation of CC's full potential.

4.3.3. "We outsource as much as possible" - Truck tracking and guiding system $\left(\boldsymbol{V}_{\boldsymbol{6}}\right)$. The case company is a global chemical player with production sites around the globe. The corporation pursues a cloud-first strategy with multiple CSPs due to two reasons. First, they want to reduce the utilization of local data centers to save on space and mitigate the risks of data centers located close to chemical production sites. Second, the company aims to achieve additional flexibility in terms of usage peaks and the rollout of software to affiliate companies in cases of mergers and acquisitions. Our interviewee works as a Global Category Buyer in strategic CSP management, focusing on $\mathrm{CC}$.

One of the larger projects currently under planning is the forecasting and scheduling of trucks entering the main facility to unload chemical components. Due to spatial limitations and legal requirements, only a certain number of trucks may be on the production site at a certain point in time, having loaded specific chemicals. Therefore, a fully automated system, tracking the lorries on the surrounding highways and parking lots, checking and issuing required paperwork, and guiding vehicles to the correct unloading stations is of significant business benefit. The company uses platform services for building this IS for multiple reasons. First, the corporation sources existing platform services like image recognition and forecasting algorithms to ideally 
steer the approaching trucks. Second, by cooperating on the same platform together with partners (e.g., providers of camera equipment), they aim to include third party knowledge and easily outsource the operations and maintenance. Third, by sourcing software components and outsourcing of significant parts of the additional development, the company intends to save on internal resources to focus on steering and the contribution of core knowledge regarding the production site and chemical considerations.

"We aim to outsource as much as possible and focus on our core competencies. For the project, we collect the needed software buckets, CSPs, and partners. We only steer the process. For the final product, we take care of the supervision and leave the maintenance to experts."

In conclusion, the company aims to reduce internal efforts as much as possible (focus on core competencies). If available, a SaaS offering would probably be the most favorable option for the case company. However, as this specialized software is not available off-the-shelf, the company uses CC to source as many available components as possible. It combines functionalities modularly on their selected platform (access to specialized resources). These include functionalities (image recognition), hardware (cameras) as well as workforce for development, operations, and maintenance. $\mathrm{CC}$, in this case, can be seen as a measure to combine advantages of conventional outsourcing with the benefits of individualization and increased steering possibilities.

\subsection{Case Vignettes on SaaS post-adoption}

4.4.1. "We save scarce human resources in IT to focus on core business activities" - HR system ( $\left.V_{7}\right)$. The company of the following case study is a global player in the automotive industry with production sites all around the world. The three main reasons for $\mathrm{CC}$ adoption are speed, innovativeness, and ease of use, whereas cost reduction is not a driver for the usage of CC. The company uses various public CSPs for different business purposes. Own IS developments aim to achieve a competitive edge and strategic advantage in the core business. Our participant is a Cloud Architect, focusing on the enterprise resource planning (ERP) transformation within the group.

The human resource (HR) system comprises functionalities as master data management, recruiting interns and new hires, the onboarding process, and talent management. The company considered shifting the HR system, which was previously hosted on-premise, to CC. Therefore, the company evaluated, despite the ongoing "war for talent," that developing HR systems did not belong to the strategic core business (strategic importance). So, the company decided to rely on SaaS to source the system instead of development on PaaS or
IaaS. Regarding reasons for migrating to $\mathrm{CC}$, the participant stated that continuously fewer services are supported in the on-premise variant of CSP's HR system, increasing the relative attractiveness of cloud-based solutions compared to the on-premise implementation. The advantages of using SaaS showed especially in the rollout of the new system:

"The rollout went tremendously fast. We could use the CSP's data centers worldwide and, therefore, easily comply with local regulations. Additionally, we could migrate existing data quickly via middleware offered along with the SaaS solution. As a result, we save scarce human resources in IT that no longer have to spend time upgrading and patching legacy HR systems but can now focus on core business."

In conclusion, this vignette shows that dominant factors supporting the usage of SaaS offerings are a fast implementation (time to market) of ready-to-use ISs (access to specialized resources) and an eased operation, that reduces the workload on digital talent. SaaS, therefore, seems to be the delivery model of choice in fields of commodity software that does not allow for strategic differentiation (focus on core competencies). Cost savings did not influence the company's decision for $\mathrm{CC}$, in this case, as the CSP's pricing scales by the number of employees included in the subscription. This pricing model distinguishes SaaS from PaaS/IaaS, where actual usage rather than headcount defines the cost basis. Migrating sensitive information to the public cloud creates a dependency on a third party regarding data security but did not hinder the case company from migrating the core data of employees to the public cloud.

4.4.2. "The internal processes took us 12 months whereas provisioning needed only four days" Digital signature system $\left(\boldsymbol{V}_{\boldsymbol{8}}\right)$. The case company is a tier-1 automotive supplier. In line with the cost-oriented business strategy, the strategic goal of CC on a company level is the reduction of cost. Our study participant acts as a Transformation Manager in the digitization office, focusing on $\mathrm{CC}$ projects for the past years.

The company implemented a digital signature system to simplify and fasten the signing of contracts with multiple suppliers. Previously, all contracts with vendors needed four physical signatures, including two internal colleagues and two representatives of the vendor. Therefore, paper-based contracts have been circularly sent to the relevant colleagues, partially across multiple continents, taking up to several weeks. This process costed significant postage and caused enormous effort amongst employees to track the status and conducting follow-ups. Things got even more complicated when the COVID-19 pandemic struck the company. Several employees, including the head of purchasing, needed to work from home without the 
necessary equipment (e.g., printer) to fulfill the required tasks. Thus, the processes became further delayed.

As a response, the company sourced a cloud-based software for digital signatures from a $3^{\text {rd }}$ party CSP. The software allows signing contracts legally entirely remotely and instantaneously by recording IP-address, mail access, and timestamp of every signature made. According to the participant, the SaaS complied with any security certifications, and the CSP could not access the content of the signed contracts due to embedded encryption. Notably, only one internal person needed a license of the program to create the contract, whereas the ones signing do not need the software. The participant worked on the preparations for piloting the application for several months, but budget constraints and internal application cycles hindered implementation. Given the crisis, the company released the budget by board approval, and from then on, things went fast:

"As soon as we had the budget allocation, we signed the contract digitally on Thursday, and the software was ready to use on Sunday. Absurd that on the one hand, it took 12 months of internal work - which was super-fast compared to other projects - and on the other hand, only four days to provision the software. Previously, it took more time than that even to sign the contract."

This case study shows that all the speed and flexibility provided by $\mathrm{CC}$ can only unfold when the corporate structures and processes do not slow down the implementation of $\mathrm{CC}$ projects. In the case company, annual budget allocations lead to up to 24 months of lead time before the start of the project, consuming a factor of a hundred times the period of the actual rollout of the software solution. Although the company's overarching goal of $\mathrm{CC}$ is to save on money, given the corona crisis for the relevant project, predominantly the speed of the provisioning of ready-to-use solution (reduced time to market) of commodity services (focus on core competencies) was of major importance. It was of consideration that the cost to sign a contract digitally is cheaper than the average postage (cost savings). However, the potential labor savings through the simplified signature process are challenging to materialize due to strong labor unions in the German automotive sector. Regarding security risks, the company believes that "thousands of security specialists" at the CSPs can develop more secure advanced systems than an individual company possibly could.

\section{Discussion and recommendations}

The reasons for $\mathrm{CC}$ delivery model post-adoption varied to some extent but also exhibited commonalities. Firstly, the below discussion derives decision criteria for selecting the appropriate CC delivery model on IS level. Secondly, findings across delivery models serve as recommendations for practitioners on capturing the benefits of $\mathrm{CC}$ on a post-adoption level.

\subsection{Decision criteria for selecting $\mathrm{CC}$ delivery models}

When discussing the usage of IaaS, reduced time to market and flexibility have been the two factors of the highest importance to all participants. These determinants show a significant advantage of cloudbased infrastructure compared to on-premise solutions when companies need to develop specific and specialized software on a scalable infrastructure. Flexibility in this manner refers to either temporary extension of underlying capacities (e.g., demand peaks $V_{l}$ ) or the permanent increase of computing power or storage (e.g., $V_{2}$ ).

Decision criteria 1: Use IaaS as a delivery model if building a custom system that possibly exhibits fluctuations in needed computing capacity.

Regarding the usage of PaaS, access to specialized resources in terms of ready-to-use functionalities and components has been the dominant determinant for delivery model selection. Using these functionalities, the study's participants mentioned the possibility to save development effort (e.g., $V_{5}$ ) and similarly to gain speed in implementation (e.g., $V_{6}$ ). Additionally, these functionalities drive innovation.

Decision criteria 2: Use PaaS to save time by leveraging ready-to-use functionalities to focus on the context-specific implementation.

Section 4.4 revealed that large-scale companies use SaaS for IS that constitute a commodity (e.g., $V_{8}$ ). In the case of the HR system, the company in $V_{7}$ faced a decision whether this IS constitutes such a commodity, or whether the company aims to build it itself to differentiate from the competition. Consequently, using SaaS spares IT development resources that can be reallocated to focus on core competencies.

Decision criteria 3: Proactively assess whether to consider an IS as a strategic differentiator - if not, use SaaS.

The decision criteria above are in line with recommendations postulated by practitioners [19]. Through the interviews and the conducted review of our decision criteria with three participants, we confirmed the decision criteria's validity on a qualitative basis.

\subsection{Learnings from IS-level post-adoption of CC}

While almost all participants considered time to market as a reason for CC post-adoption (consistent with the literature [5] on $\mathrm{CC}$ adoption), this expression 
reveals different meanings depending on the delivery model. In $\mathrm{V}_{8}$, the company considered the implementation as fast because the rollout on a global scale could be conducted quickly. For PaaS and IaaS, the adoption of the CSP itself was a project of several months, according to $\mathrm{V}_{2}$. The post-adoption to bring new use-cases on IaaS is fast according to $\mathrm{V}_{l}$ because the service provisioning is available instantly. Interviewees considered PaaS post-adoption as fast because the functionalities did not have to be built (e.g., $\mathrm{V}_{4}$ ) individually. While the provisioning of IaaS and PaaS was within seconds, and for SaaS within days, we see the time-limiting factor in beforehand budget discussions, proposal processes, worker council approvals, and IT security evaluations:

Recommendation I: Ensure that governance does not inhibit the benefits of time to market from $\mathrm{CC}$.

Analyzing concrete post-adoption implementations of $\mathrm{CC}$, we could not identify the factor of cost advantages across delivery models. This finding is different from previous studies [5] that primarily regarded the initial adoption of $\mathrm{CC}$ on the company level. While four out of eight interviewees stated that their companies target the realization of cost advantages through CC adoption on a company level, none of the vignettes revealed cost considerations as a core factor for the individual IS in post-adoption. We hypothesize two (non-alternative) explanations: Firstly, cost advantages come through company-level decisions. These could be contracting multiple CSPs or data center reduction. Secondly, cloud-based services potentially allow for labor savings due to simplified operations and maintenance. However, IT-specialists are often not discharged but reallocated to alternative projects:

Recommendation II: Capture cost savings on a company level if this is a motivation for $\mathrm{CC}$ adoption.

Contrary to the literature on CC and IT outsourcing [5], we find that the strategic importance of the IS positively influences most post-adoption decisions of IaaS and PaaS. We attribute this change from a negative to a positive relationship to $\mathrm{CC}$ becoming the state-ofthe-art standard for IS development, as identified in our pilot study by one participant [2, p. 11]: "I believe our future competitive advantages will be based on CC." Recommendation III: Consider CC also for strategically important ISs.

\subsection{Limitations}

The applicability of our findings is limited to the context and research methodology used to obtain the findings. Regarding the context, we acknowledge that our findings are limited to the scope of large-scale companies, as the reasons for post-adopting CC delivery models might differ for smaller companies or academic institutions. Furthermore, we conducted our study with companies headquartered in Germany, so the results apply to developed countries. Additionally, the interviewed companies do not fulfill the requirements of representative sampling, although we paid attention to select interview partners from multiple industries. Regarding the research methodology, we acknowledge that our findings rely on purely qualitative data and yet require further quantitative research to test its generalizability in and beyond the scope of large-scale companies in developed countries. The above-stated recommendations serve as guidance for delivery model selection but do not guide whether an IS should be implemented with CC technology.

\section{Conclusion}

The paper at hand investigates three RQs: Firstly, we observe that the determinants of IS-level CC implementations vary by delivery model. Companies implement IaaS mostly for flexibility, PaaS for access to specialized resources, and $S a a S$ to keep focus on core competencies. Secondly, we derive decision criteria for CC delivery model selection based on our findings. Thirdly, we elaborate on the learnings from ISlevel implementations regarding cost advantages, timeto-market, and strategic importance. These learnings partly contradict current findings in the literature focusing on company-level adoption of CC.

We see two further avenues for research based on the findings of this paper: Firstly, we see the opportunity to postulate a research model to test findings in a quantitative research setting, as proposed by Gable [12] for the integration of qualitative and quantitative research. Secondly, our interviews surfaced that the corporate usage of $\mathrm{CC}$ and the strategy it follows show different manifestations in companies. Therefore, we see the potential to investigate the building blocks and dimensions of corporate $\mathrm{CC}$ strategies in further studies.

\section{References}

[1] A. Pols and M. Vogel, "Cloud-Monitor 2019," Bitkom, 2019 https://www.bitkom.org/sites/default/files/201906/bitkom_kpmg_pk_charts_cloud_monitor_18_06_20 19.pdf.

[2] F. Wulf, M. Westner, M. Schön, S. Strahringer, and C. Loebbecke, "Preparing for a Digital Future: Cloud Strategy at Continental AG," In ICIS 2019 Proceedings, 2019.

[3] F. Bagayogo, L. Lapointe, and G. Bassellier, "Enhanced Use of IT: A New Perspective on Post-Adoption," JAIS, vol. 15, no. 7, 2014, pp. 361-387.

[4] P. Mell and T. Grance, "The NIST Definition of Cloud Computing," 2011.

[5] S. Schneider and A. Sunyaev, "Determinant Factors of Cloud-Sourcing Decisions: Reflecting on the IT Outsourcing Literature in the Era of Cloud Computing," 
Journal of Information Technology, vol. 31, no. 1, 2016, pp. 1-31.

[6] M. Moeini, Y. Rahrovani, and Y. E. Chan, "A Review of the Practical Relevance of IS Strategy Scholarly Research," The Journal of Strategic Information Systems, vol. 28, no. 2, 2009, pp. 196-217.

[7] "MIS Quarterly Executive Mission and Scope," AIS eLibrary. https://aisel.aisnet.org/misqe/aimsandscope.html.

[8] R. Seethamraju, "Determinants of SaaS ERP Systems Adoption," in PACIS 2013 Proceedings, 2013, pp. 116.

[9] A. Benlian, T. Hess, and P. Buxmann, "Drivers of SaaSAdoption - An Empirical Study of Different Application Types," Business \& Information Systems Engineering, vol. 1, no. 5, 2009, pp. 357-369.

[10] A. Benlian and T. Hess, "ERP Software as Service (SaaS): Factors Affecting Adoption in South Africa," Decision Support Systems, vol. 52, no. 1, 2011, pp. 232-246.

[11] M. Lechesa, L. Seymour, and J. Schuler, "ERP Software as Service (SaaS): Factors affecting adoption in South Africa," in Re-conceptualizing Enterprise Information Systems, Springer, Heidelberg, Germany, 2011, pp. $152-167$.

[12] G. G. Gable, "Integrating Case Study and Survey Research Methods: An Example in Information Systems," European Journal of Information Systems, vol. 3, no. 2, 1994, pp. 112-126.

[13] S. L. Pan and B. Tan, "Demystifying Case Research: A Structured-Pragmatic-Situational (SPS) Approach to Conducting Case Studies," Information and Organization, vol. 21, no. 3, 2011, pp. 161-176, Nov. 2011.

[14] M. C. Lacity, L. P. Willcocks, and S. Khan, "Beyond Transaction Cost Economics: Towards an Endogenous Theory of Information Technology Outsourcing," The Journal of Strategic Information Systems, vol. 20, no. 2, 2011, pp. 139-157.

\section{Appendix}

\section{Determinant definitions}

Cost savings - "Total cost advantage of sourcing IT resources from an external vendor compared with the costs for alternative provisioning (e.g., in-house). Total costs comprise transaction costs and production costs." [5, p. 28]

Strategic importance - "The degree of strategic value that companies attach to an asset [9]. Assets of high strategic importance create and exploit unique sources of value [20] and enable organizations to sustain a competitive advantage [21]." [5, p. 29]

Access to specialized resources - "Client's benefit from economies of skill by leveraging the skills, resources, and capabilities that the vendor offers (e.g., access to the latest technologies and IT-related know-
[15] M. C. Lacity, S. Khan, A. Yan, and L. P. Willcocks, "A Review of the IT Outsourcing Empirical Literature and Future Research Directions," Journal of Information Technology, vol. 25, no. 4,2010, pp. 395-433.

[16] E. Niemimaa and M. Niemimaa, "Information Systems Security Policy Implementation in Practice: From Best Practices to Situated Practices," European Journal of Information Systems, vol. 26, no. 1, 2017, pp. 1-20.

[17] C. E. Huang Chua and M. D. Myers, "Social Control in Information Systems Development: A Negotiated Order Perspective," Journal of Information Technology, vol. 33, no. 3, 2018, pp. 173-187.

[18] J. Kotlarsky, H. Scarbrough, and I. Oshri, "Coordinating Expertise Across Knowledge Boundaries in OffshoreOutsourcing Projects: The Role of Codification," $M I S Q$, vol. 38, no. 2, 2014, pp. 607-627.

[19] M. J. Kavis, Architecting the Cloud - Design Decisions for Cloud Computing Service Models (SaaS, PaaS, IaaS). John Wiley \& Sons, Hoboken, New Jersey, 2014.

[20] C. Loebbecke and C. Huyskens, "What Drives Netsourcing Decisions? An Empirical Analysis," European Journal of Information Systems, vol. 15, no. 4, 2006, pp. 415-423.

[21] B. Watjatrakul " Determinants of IS sourcing decisions: A Comparative Study of Transaction Cost Theory Versus the Resource-Based View," The Journal of Strategic Information Systems, vol 14, no. 4, 2005, pp.389-415.

[22] S. Saya, L. G. Pee, and A. Kankanhalli, "The Impact of Institutional Influences on Perceived Technological Characteristics and Real Options in Cloud Computing Adoption," In ICIS, 2010.

[23] S. Subashini and V. Kavitha, "A Survey on Security Issues in Service Delivery Models of Cloud Computing," Journal of Network and Computer Applications, vol. 34, no. 1, 2011, pp. 1-11.

how). These specialized capabilities could not be generated internally [10]." [5, p. 29]

Flexibility - "The benefits of increased flexibility due to the scalable, on-demand, and pay-per-use provisioning of IT resources and the trialability of services [10, 22]." [5, p. 29]

Focus on core competences - "The organization's ability to focus on core business activities (e.g., [10])." [5, p. 29]

Reduced time to market - "The organization's ability to deliver its products or services faster to the market when sourcing services externally (e.g., [8])." [5, p. 30]

Security risk - "Security risks associated with remote data hosting, virtualized and shared resources, and data transfer over the Internet [23]." [5, p. 30] 